\title{
Type II cGMP-dependent protein kinase inhibits EGF-induced MAPK/JNK signal transduction in breast cancer cells
}

\author{
TING LAN, YONGCHANG CHEN, JIANRONG SANG, YAN WU, YING WANG, LU JIANG and YAN TAO \\ School of Medical Science and Laboratory Medicine, Jiangsu University, Zhenjiang, Jiangsu, P.R. China
}

Received November 24, 2011; Accepted February 7, 2012

DOI: 10.3892/or.2012.1726

\begin{abstract}
Our previous research data showed that type II cGMPdependent protein kinase (PKGII) inhibited EGF-induced MAPK/ERK-mediated signal transduction through blocking the phosphorylation of EGFR caused by EGF. Since EGFR also mediates other MAPK-mediated signal transduction pathways, this study was designed to investigate whether PKGII inhibits EGF-induced MAPK/c-Jun N-terminal kinase (JNK) signal transduction. MCF-7 human breast cancer cells were infected with adenoviral constructs encoding the cDNA of PKGII (pAd-PKGII) to increase the expression of PKGII and treated with 8-pCPT-cGMP to activate the enzyme. Western blotting was applied to detect the phosphorylation/activation of EGFR, JNK, MKK7 and c-Jun. The Pull-down method was used to detect the activation of Ras protein. Co-IP was used to analyze the binding between Grb2 and Sos 1. TUNEL staining was used to detect the apoptosis of MCF-7 cells. The results showed that EGF treatment increased the phosphorylation of EGFR, the binding between Grb2 and Sos1, the activation of Ras, and the phosphorylation/activation of MKK7, JNK and c-Jun, but decreased the apoptosis of the cells. Increase of PKGII activity through infection with pAd-PKGII and stimulation with 8-pCPT-cGMP efficiently reversed the above changes caused by EGF. The results suggest that PKGII also inhibits EGF-induced MAPK/JNK-mediated signal transduction and further confirmed that PKGII can block the activation of EGFR.
\end{abstract}

\section{Introduction}

c-Jun N-terminal kinase (JNK), also known as stress-activated protein kinase (SAPK), is a member of MAPK family. JNK can be activated by a variety of environmental stresses, inflammatory cytokines, growth factors and GPCR agonists (1). Like other MAPK-mediated signal transduction pathway, MAPK/

Correspondence to: Professor Yongchang Chen, Institute of Medical Science, Jiangsu University, Zhenjiang, Jiangsu, P.R. China E-mail: ycchen54@ujs.edu.cn

Key words: type II cGMP-dependent protein kinase, MAPK/JNK, signal transduction, apoptosis, breast cancer cells
JNK-mediated pathway has a cascade reaction of kinases, including MAPKKK, MAPKK and MAPK/JNK (2). MAPKK in this pathway includes MKK4 and MKK7 which can activate JNK through causing dual phosphorylation of this kinase (3). In the upstream of MAPKK, there are several kinases which can be grouped into MAPKKK, including MAPK/ERK kinase (MEKK) 1, 2, 3, 4, apoptosis signal-regulating kinase (ASK), mixed lineage kinases (MLKs) and TGF- $\beta$ activated protein kinase (TAK). In MAPK/JNK-mediated signal transduction pathway, the main MAPKKK is MEKK1 (4). MEKK1 can be activated by active Ras GTPase, a key component of MAPK-mediated pathway. Through the mediation of adaptor proteins such as Grb2 and Sos1, Ras activation is linked to several growth factor receptors, and EGFR is one of them. In the downstream of the pathway, activated JNK can be translocated to the nucleus where it regulates the activity of multiple transcription factors, and c-Jun is one of them $(5,6)$. Apoptosis is one of the biological activities of the cells regulated by activated JNK $(7,8)$. In the regulation of apoptosis, JNK functions as double-edged sword, depending on cell type, nature of the death stimulus, duration of its activation and the activity of other signaling pathways (9).

cGMP-dependent protein kinases (PKGs) are serine/ threonine kinases and currently two types of PKGs have been indentified in mammalian cells: type I cGMP-dependent protein kinase (PKGI) and type II cGMP-dependent protein kinase (PKGII) $(10,11)$. One of the new findings in PKG research is the association of the kinases with proliferation of cells, especially tumor cells. Research data revealed that PKGI could suppress the growth of tumor cells and this kinase has been identified as a tumor suppressor (12). Recently, we found that the expression and the activity of PKGII in human gastric cancer cell lines were significantly lower than that in normal gastric mucosal cells and increasing PKGII activity through infecting the cancer cells with adenovirus victor encoding PKGII cDNA and stimulating the cells with cGMP analog 8-pCPT-cGMP inhibited the proliferation of gastric cancer cell lines (13). Further study in our laboratory revealed that the inhibitory effect of PKGII on EGF-induced proliferation of cancer cell was related to the suppressive effect of PKGII on MAPK/ERK-mediated signal transduction pathway and the blocking point was the activation of EGFR (14). Since EGFR also initiates other MAPK-mediated signal pathways such MAPK/JNK-mediated pathway (15), it deserves further investigation whether PKGII has inhibitory effect on other 
MAPK mediated signal pathways except MAPK/ERK mediated pathway. This study was designed to elucidate the possible inhibition of PKGII on MAPK/JNK-mediated signal transduction pathway.

\section{Materials and methods}

Antibodies, chemicals and cell lines. Antibodies against pEGFR (Tyr1068) and pMKK7 (Ser271/Thr275) were purchased from Cell Signaling (Danvers, MA, USA). Antibodies against Sos1, Grb2, pan-Ras and $\beta$-actin were from Santa Cruz Biotechnology, Inc. (Santa Cruz, CA, USA). Antibodies against pJNK1/2/3(Thr183+Tyr185) and p-c-Jun(Ser73) were from Bioworld Technology (St. Louis Park, MN, USA). Antibody against PKGII was from ABGENT Biotechnology (San Diego, CA,USA).Horseradish peroxidase (HRP)-conjugated secondary antibodies were from Jackson Immuno-Research Laboratories (West Grove, PA, USA). Electrochemiluminescence (ECL) reagents were from Millipore (Billerica, MA, USA). Dulbecco's modified Eagle's medium (DMEM) and new-born calf serum (NBCS) were from Gibco (Grand Island, NY, USA).

Human breast cancer cell line MCF-7, adenoviral vectors encoding cDNA of $\beta$-galactosidase (pAd-LacZ) and PKGII (pAd-PKGII) were kind gifts from Dr Gerry Boss and Dr Renate Pilz in University of California, San Diego, USA. In Situ Cell Death Detection kit (TUNEL Technology) was from Roche (Roche Diagnostics, Mannheim, Germany).

Western blotting. Protein samples were subjected to SDS-PAGE (8-12\%) gel according to the molecular size of target protein, and electrophoresis and membrane transfer was performed following the manufacturer's protocol (Bio-Rad, Hercules, CA, USA). The primary antibodies were incubated overnight at $4^{\circ} \mathrm{C}$ in TBS-T (2\% Tween-20), and the corresponding secondary antibodies were incubated for $1 \mathrm{~h}$ at RT in TBS-T ( $2 \%$ Tween-20), with three washes after each incubation. ECL reagents were used to show the positive bands on the membrane.

Ras activity assay. The activity of Ras was detected with Pull-down method as described previously (16). In brief, cells growing on $100-\mathrm{mm}$ culture plate were washed three times with cold PBS and lysed by adding $400 \mu 1$ of the lysis buffer (25 mM HEPES pH 7.5, $150 \mathrm{mM} \mathrm{NaCl}, 1 \%$ NP40, $10 \%$ glycerol, $25 \mathrm{mM} \mathrm{NaF}, 10 \mathrm{mM} \mathrm{MgCl} 2,0.25 \%$ sodium deoxycholate, $1 \mathrm{mM}$ EDTA, $1 \mathrm{mM} \mathrm{Na}_{3} \mathrm{VO}_{4}, 10 \mu \mathrm{g} / \mathrm{ml}$ aprotinin, and $10 \mu \mathrm{g} / \mathrm{ml}$ leupeptin). The sample was collected and centrifuged $\left(14,000 \mathrm{xg} / \mathrm{min}, 4^{\circ} \mathrm{C}, 10 \mathrm{~min}\right)$ to remove the debris. The supernatant was incubated with glutathione-Sepharose beads and glutathione S-transferase-Ras-RBD (GST-RBD) at $4^{\circ} \mathrm{C}$ for $1 \mathrm{~h}$. The beads were washed 3 times with lysis buffer and heated in boiled water to release proteins. The protein samples (containing active Ras) were analyzed by western blotting with antibody against pan-Ras.

Co-immunoprecipitation. The cells growing on 100-mm culture plate were washed two times with cold PBS and lysed by adding $1 \mathrm{ml}$ RIPA buffer (50 mM Tris-HCl pH 7.4, $1 \%$ Triton X-100, $1 \mathrm{mM}$ EDTA, $1 \mathrm{mM}$ leupeptin, $1 \mathrm{mM}$ phenylmethylsulfonyl fluoride, $10 \mathrm{mM} \mathrm{NaF}, 1 \mathrm{mM} \mathrm{Na} \mathrm{VO}_{4}$ ) per plate. Antibodies against Grb2 and Sos1 and isotype matched IgG were used for immunoprecipitation.

Detection of apoptosis. Terminal deoxynucleotidyl transferasemediated dUTP nick end-labeling (TUNEL) was performed with In Situ Cell Death Detection kit, following the manufacturer's instructions. Briefly, cells grown on 24 well the plate were fixed and endogenous peroxidase activity was quenched with $2 \% \mathrm{H}_{2} \mathrm{O}_{2}$ for $5 \mathrm{~min}$. The cells were incubated with reaction buffer (containing terminal deoxynucleotidyl transferase (TdT) buffer, TdT, $1 \mathrm{mM} \mathrm{Mn}^{2+}$, and fluorescein-labled dUTP) in a humid atmosphere at $37^{\circ} \mathrm{C}$ for $60 \mathrm{~min}$. The cells were washed with PBS and incubated with anti-fluorescein antibody conjugated with horseradish peroxidase for $30 \mathrm{~min}$. After rinsing with PBS, the cells were immersed in DAB solution. Under microscope, cells exhibiting brown nuclear staining were taken as apoptotic cells. For each well, five x200 fields were randomly selected and the positive cells in the fields were counted. The average of the positive cell numbers was taken as value of one experiment. The assay was repeated three times.

\section{Results}

PKGII prevents EGF-induced Tyr1068 phosphorylation of EGFR. When EGF binds with EGFR, tyrosine 1068 (Y1068) is one of the auto-phosphorylation sites of EGFR. Phosphorylation of this site is associated with MAPKmediated signaling. In this experiment, we investigated the inhibitory effect of PKGII on the phosphorylation of EGFR (Y1068) in differently treated MCF-7 cells by using western blotting. The results showed that EGF treatment caused an obvious increase of Y1068 phosphorylation of EGFR. In cells infected with pAd-PKGII and stimulated with cGMP, the phosphorylation was significantly decreased (Fig. 1). This indicated that PKGII prevented the Y1068 phosphorylation of EGFR caused by EGF.

PKGII inhibits the binding between Grb2 and Sos1. Grb2 is a growth factor receptor-bound protein and Sos1 is a guanine nucleotide exchange factor (GEF). Both of them play a role of adaptor protein in MAPK signal pathway. Once they bind each other, the downstream signal component Ras is activated. Co-IP method was applied to detect the binding between these two proteins and the effect of different treatment on the binding. The results showed that EGF treatment increased the binding between Grb2 and Sos1. In the cells infected with pAd-PKGII, stimulated with cGMP and then treated with EGF, the binding level between two proteins was much lower than that of cells infected with pAd-LacZ and treated with EGF only (Fig. 2). These results revealed that PKGII inhibited the EGF-induced binding between Grb2 and Sos1.

PKGII prevents the activation of Ras and the phosphorylation of MKK7. Same as in MAPK/ERK and MAPK/p38 mediated signal pathways, small $\mathrm{G}$ protein Ras is also the key component of the MAPK/JNK mediated pathway. Ras has two forms in cells: GTP-bound activated form and GDP-bound inactivated form. Once Ras is the GTP-bound form, it activates downstream signal component and guides a series of activations of kinases in the signal pathway. In this experiment, the Pull-down method 


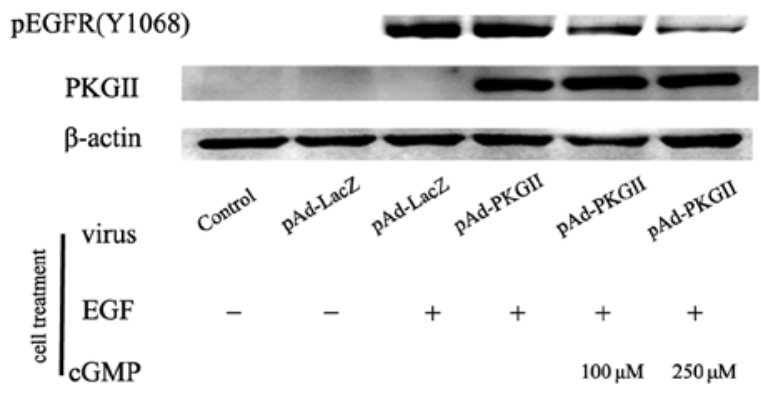

Figure 1. PKGII inhibits EGF-induced Y1068 phosphorylation of EGFR MCF-7 cells were infected with pAd-LacZ or pAd-PKGII for $48 \mathrm{~h}$ and serum starved $\mathrm{o} / \mathrm{n}$. In LacZ+EGF and PKGII+EGF groups, cells were incubated with EGF (100 ng/ml) for $5 \mathrm{~min}$. In pAd-PKGII+cGMP+EGF groups, cells were treated with 8-pCPT-cGMP for $1 \mathrm{~h}$ and then with EGF $(100 \mathrm{ng} / \mathrm{ml})$ for $5 \mathrm{~min}$. Cells were harvested and lysed as described in Materials and methods and cell lysate was subjected to western blotting. The results showed that the infection with pAd-PKGII caused obvious increase of PKGII expression. EGF treatment induced dramatic increase of phosphorylation of EGFR. Infection with pAd-PKGII+cGMP treatment efficiently inhibited the EGFinduced phosphorylation of EGFR.

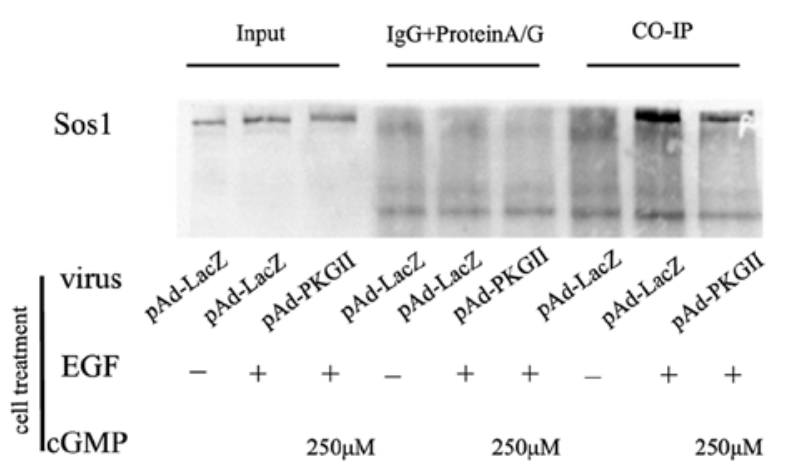

Figure 2. EGF induced binding between Grb2 and So-s1 and PKGII inhibited the binding. MCF-7 cells were grown in 100-mm plates and infected with either pAd-LacZ or pAd-PKGII. Then, the cells were serum-starved overnight and treated differently: in pAd-LacZ group, no drug treatment; in pAd-LacZ+EGF group, the cells were incubated with EGF $(100 \mathrm{ng} / \mathrm{ml})$ for $5 \mathrm{~min}$; in pAd-PKGII+cGMP+EGF group, cells were incubated with with $250 \mu \mathrm{M} 8$-pCPT-cGMP for $1 \mathrm{~h}$ and followed by incubating with EGF $(100 \mathrm{ng} / \mathrm{ml})$ for $5 \mathrm{~min}$. Co-immunoprecipitation was performed to analyze the effect of EGF and PKGII on the binding between Grb2 and Sos1. The results showed that in EGF-treated cells, the binding level between Grb2 and Sos1 was much higher than that in untreated cells. Higher expression and activity of PKGII significantly inhibited the binding.

was applied to detect EGF-induced activation of Ras and the effect of PGKII on the activation. The results showed that EGF treatment induced an obvious increase of GTP-bound Ras and increase of PKGII activity effectively prevented EGF-induced Ras activation (Fig. 3).

MKK7 is a MAPKK downstream of Ras and serves as a specific activator of JNK. During the signal transduction process, active Ras can activate MEKK1, the MAPKKK in MAPK/JNK pathway, and MEKK1 can phosphorylate Ser271/ Thr275 of MKK4/MKK7, the MAPKKs in this pathway. In this study, western blotting was applied to detect the Ser271/ Thr275 phosphorylation of MKK7. The results showed that the level of pMKK7 (Ser271/Thr275) increased obviously in cells infected with pAd-LacZ and treated with EGF, and was much lower in cells infected with pAd-PKGII, stimulated with cGMP and then treated with EGF (Fig. 4).

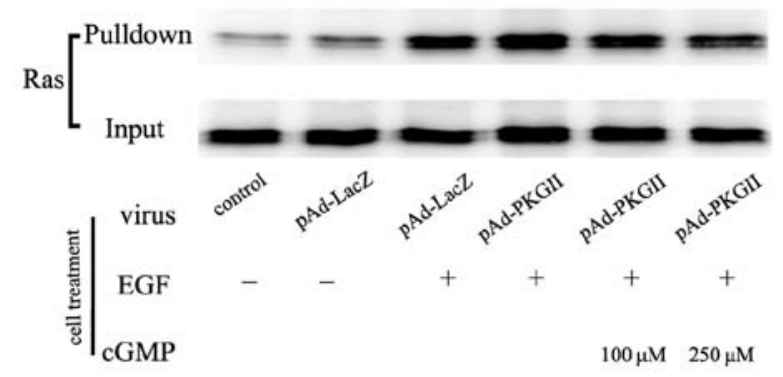

Figure 3. PKGII inhibits EGF-induced Ras activation. The Pull-down method was used to detect the activated Ras. Cell lysate was prepared and equal amounts of protein were incubated with GST-RBD beads as described in Materials and methods. Binding complexes were collected by centrifugation, resolved by SDS-PAGE, and probed with anti-pan Ras antibody. The results showed that in cells infected with pAd-LacZ, EGF treatment (pAdLacZ+EGF) caused an obvious activation of Ras. In cells infected with pAd-PKGII, pre-incubation with 8-pCPT-cGMP for $1 \mathrm{~h}$ (pAd-PKGII+cGMP) inhibited EGF-induced Ras activation. The results shown are representative of three separate experiments.

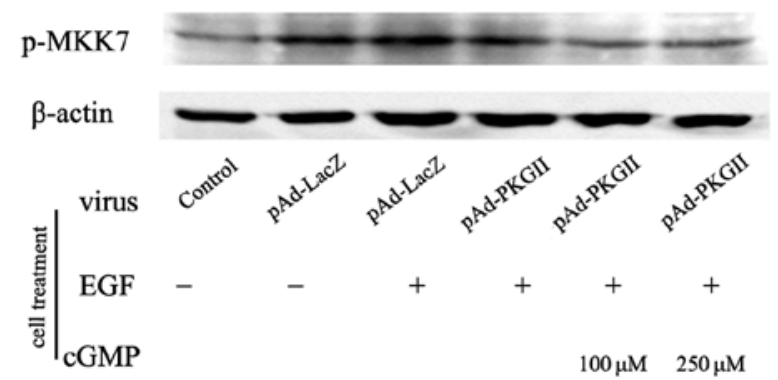

Figure 4. PKGII prevents EGF-induced MKK7 phosphorylation. MCF-7 cells were infected with pAd-LacZ or pAd-PKGII for $48 \mathrm{~h}$. The cells were serum starved overnight and treated differently: in pAd-LacZ+EGF and pAdPKGII+EGF groups, cells were incubated with EGF (100 ng/ml) for $5 \mathrm{~min}$; in pAd-PKGII+cGMP groups, cells were incubated with 100 and $250 \mu \mathrm{M}$ 8-pCPT-cGMP, respectively, for $1 \mathrm{~h}$, and then incubated with EGF for $5 \mathrm{~min}$. The cell lysate was prepared and western blotting was applied to detect the phosphorylation of MKK7. The results showed that the levels of phosphorylated MKK7 (Ser271/Thr275) in pAd-LacZ-infected and EGF-treated cells was higher than that of pAd-LacZ-infected cells. In cells infected with pAd-PKGII, treated with 8-pCPT-cGMP, and then treated with EGF, the phosphorylation level was much lower than that of pAd-LacZ-infected and EGF-treated cells.

The phosphorylation of JNK is prevented by activated PKGII. In MAPK/JNK-mediated signal transduction pathway, Thr183 and Tyr185 phosphorylation of JNK caused by MEKK is a crucial event. In this study, western blotting with antibodies against JNK and p-JNK (Thr183/Tyr185) was applied to detect the phosphorylation of JNK in EGF-treated MCF-7 cells and the effect of PKGII on this process. Results showed that EGF treatment caused an obvious increase of Thr183/Tyr185 phosphorylation of JNK and the increase of PKGII activity through infecting the cells with pAd-PKGII and stimulating the cells with cGMP efficiently prevented the EGF-induced phosphorylation/activation of JNK (Fig. 5).

The activated PKGII negatively regulates the phosphorylation of c-Jun (Ser73). c-Jun is a member of the Jun family and acts as a component of the transcription factor AP-1 in regulating gene expression. As a target protein of JNK, it can be phosphorylated by this kinase and gains activity. We applied western blotting with antibodies against phosphorylated c-Jun to detect 


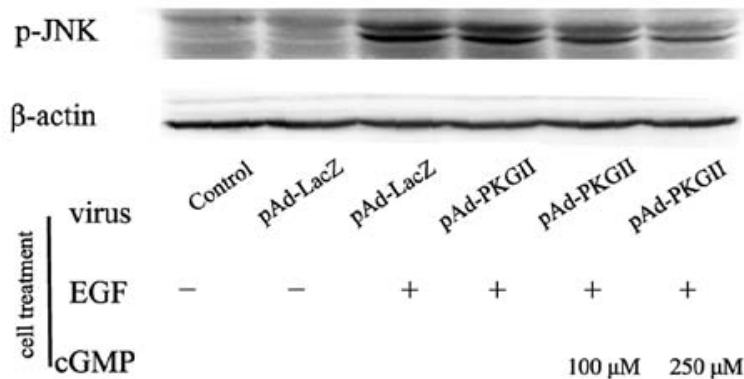

Figure 5. PKGII prevents EGF-induced JNK phosphorylation. MCF-7 cells were treated as described in Fig. 4. Western blotting was applied to detect the phosphorylation of JNK. The results showed that pAd-LacZ infection and EGF treatment caused an obvious increase of phosphorylation of JNK. Infection with pAd-PKGII and pre-treatment with cGMP before the stimulation with EGF efficiently inhibited the phosphorylation of JNK.

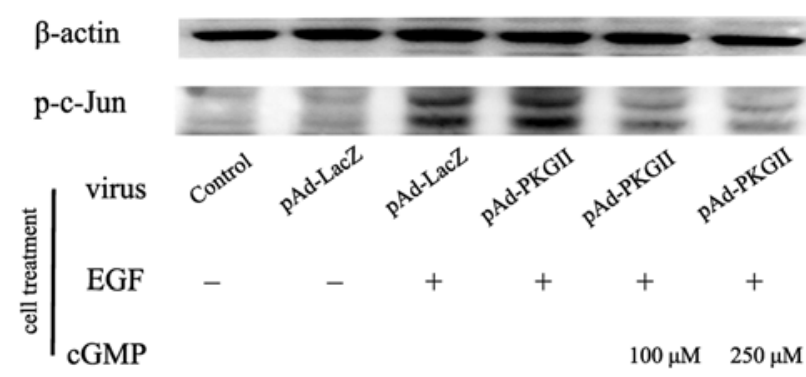

Figure 6. PKGII inhibits EGF-induced phosphorylation of C-Jun. MCF-7 cells were treated as described in Fig. 4. Western blotting was applied to detect the phosphorylation of C-Jun. The results showed that increase of PKGII activity through infecting the cells with pAd-PKGII and stimulating the cells with 8-pCPT-cGMP significantly inhibited the phosphorylation of C-Jun caused by EGF treatment.

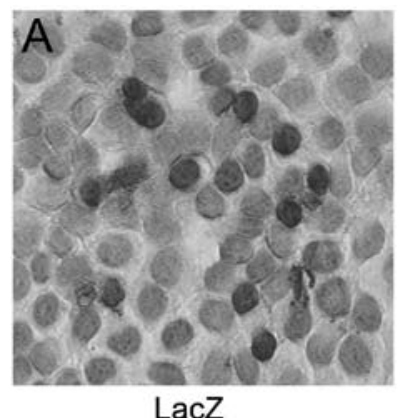

LacZ

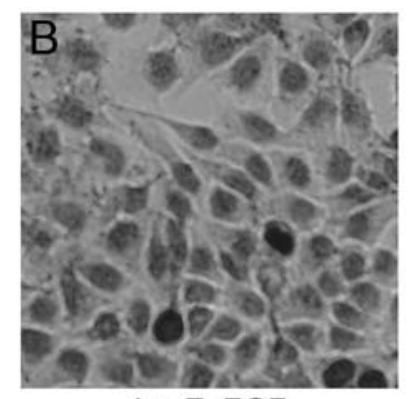

LacZ+EGF

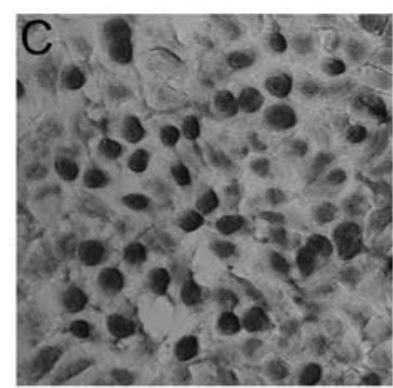

PKGII+EGF+cGMP

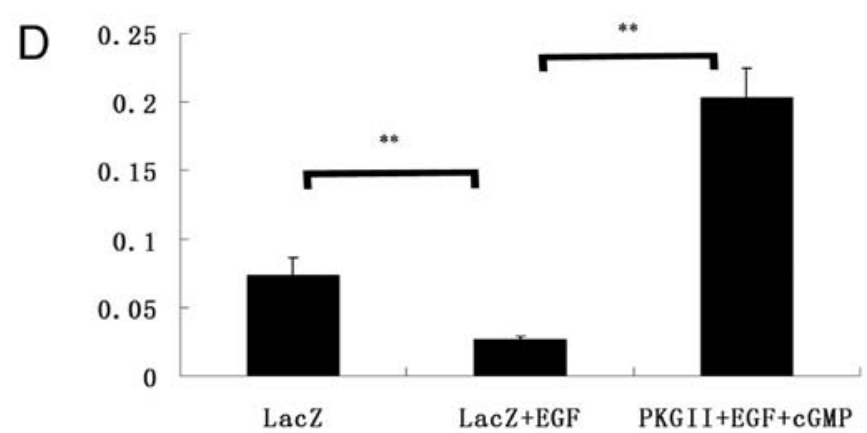

Figure 7. PKGII reverses the apoptosis-reducing effect of EGF. TUNEL staining was performed according to the manufacturer's instructions of an In Situ Cell Death Detection kit. Cells with dark brown nuclear staining were taken as apoptotic cells and counted as described in Materials and methods. (A-C) Representative images of TUNEL staining. (A) Cells were infected with pAd-LacZ only; (B) cells were infected with pAd-LacZ and inbuted with EGF (100 ng/ $\mathrm{ml}$ ) for $5 \mathrm{~min}$; (C) cells were infected with pAd-PKGII, incubated with $250 \mu \mathrm{M} 8 \mathrm{pCPT}$-cGMP for $1 \mathrm{~h}$ and then incubated with $100 \mathrm{ng} / \mathrm{ml}$ EGF for $5 \mathrm{~min}$. (D) The number of apoptotic cells per $\mathrm{x} 200$ field (the results represent mean $\pm \mathrm{SD} ;{ }^{*} \mathrm{P}<0.01$ compared with the pAd-LacZ+EGF group). The results showed that EGF treatment obviously decreased the apoptosis of MCF-7 cells while pre-treatment with $250 \mu \mathrm{M}$ 8pCPT-cGMP efficiently reversed the effect of EGF.

the phosphorylation/activation of c-Jun in EGF treated MCF-7 cells and the effect of PKGII on this process (Fig. 6). The results showed that in EGF stimulated MCF-7 cells, the level of phosphor-c-Jun increased obviously and PKGII inhibited the phosphorylation/activation of c-Jun.

The activated PKGII induced apoptosis of MCF-7 cells. One of the biological functions of MAPK/JNK-mediated signal transduction is the regulation of apoptosis of cells. In this study, TUNEL method was applied to investigate the effect of EGF and PKGII on apoptosis of MCF-7 cells. The results showed that EGF treatment reduced apoptosis of MCF-7 cells and high expression and activity of PKGII significantly increased the apoptosis of the cells, reversing the effect of EGF treatment (Fig. 7).

\section{Discussion}

As a structurally and functionally distinct enzyme, PKGII has been typically implicated in several physiological functions including intestinal secretion, bone growth, and learning and memory (17). However, recent data show that this kinase has some new functions: Nie et al (18) found that PKGII regulated epithelial sodium channels; Rangaswami et al $(19,20)$ reported that PKGII had an important role in mechano-transduction of osteoblast. A more important finding in research of PKGII is that this kinase has a role in regulating proliferation and apoptosis of cells and is potentially associated with tumorigenesis. For example, Cook et al (21) found that in cultured human prostate cells, PKGII agonist 8-pCPT-cGMP inhibited the 
proliferation induced by fetal calf serum. They also found that activation of PKGII could induce apoptosis of human prostate cells. In 2009, Swartling et al (22) reported that PKGII inhibited the proliferation of human neuroglioma cells and the inhibition was related to the decrease of the expression of transcription factor Sox 9 and the phosphorylation of Akt. In 2011, Fallahian et al (23) reported that cGMP could induce apoptosis of breast cancer cells and this effect was related to PKGII. In our laboratory, the proliferation inhibitory effect of PKGII has been confirmed by several methods including MTT assay, ${ }^{3} \mathrm{H}$-labeled thymidine uptake assay, anchorageindependent growth assay, and tumor formation in nude mouse (13). For the mechanism of the inhibition, we found that PKGII could suppress EGF-induced MAPK/ERK-mediated signal transduction. Of note, our results revealed that PKGII suppressed the signal transduction of the pathway through blocking the activation of EGF receptor, the starting event of the signal transduction process (14).

Activation of EGFR can start the signal transduction of several pathways, including MAPK-mediated pathway, PI3K/ Akt-mediated pathway, PLC $\gamma 1 / \mathrm{IP}_{3} / \mathrm{DAG}$-mediated pathway, and JAK1/STAT-mediated pathway (24-26). The signal transductions of these pathways are related not only to proliferation, but also to apoptosis and motility/migration of the cells. Does PKGII also inhibit the signal transduction of other pathways except the MAPK/ERK pathway and affect other cellular activities except proliferation? Study on these aspects are required to further elucidate the cancer suppression effect and the related mechanism of PKGII. In this experiment, we found that PKGII also suppressed the MAPK/JNK-mediated signal transduction pathway. This further confirmed the inhibitory effect of PKGII on EGF-induced activation of EGFR.

Research data have shown that JNK is a key regulator of apoptosis. JNK activation contributes to TNF- $\alpha$ and UVinduced apoptosis but can suppress apoptosis phosphorylation of the pro-apoptotic $\mathrm{Bcl}-2$ family protein $\mathrm{BAD}$, functioning as a double-edged sword in regulating apoptosis (9). In our experiment, we found that EGF treatment prevented the apoptosis of MCF-7 cells, and PKGII could reverse the antiapoptosis effect of EGF. This suggests that PKGII not only inhibits the proliferation but also induces the apoptosis of cancer cells and both effects are related to the blocking action of PKGII on EGFR activation.

EGFR is closely associated with tumorigenesis. Overexpression and mutation of EGFR occurs in most cancers (27). In vitro experiments confirmed that overexpression of EGFR caused transformation of NIH-3T3, Rat-1 and NRK cells and blocking EGFR activation inhibited proliferation of some tumor cells (28). Furthermore, a clinical study shows that cancer patients with overexpression of EGFR usually have poor prognosis. For example, EGFR overexpression was detected in $60 \%$ of non-small cell lung cancer (NSCLC) patients and the prognosis of the patients were poor, with a survival of 4-5 months (29). Therefore, EGFR is a potential cancer therapy target and methods of inhibiting EGFR activity and related signal transduction, including specific antibodies against EGFR and inhibitors of EGFR, have been intensely studied (30). Our results show that PKGII can inhibit the activation of EGFR, the consequent signal transductions and the related biological activities such prolif- eration and apoptosis, providing new clues to the strategy of cancer therapy and strongly suggesting that PKGII is a cancer suppression factor.

\section{Acknowledgements}

This study was supported by the National Natural Science Foundation of China, nos. 31040002, 81001100 and 31100974; the Innovation Grant of Jiangsu University; the Specialized Research Fund for Senior Personnel Program of Jiangsu University no. 11JDG032. We thank Dr Gerry Boss and Dr Renate Pilz, University of California, San Diego, USA for the kind gifts of Adenoviral constructs and MCF-7 cell line.

\section{References}

1. Dougherty CJ, Kubasiak LA, Frazier DP, Li H, Xiong WC, Bishopric NH and Webster KA: Mitochondrial signals initiate the activation of c-Jun N-terminal kinase (JNK) by hypoxiareoxygenation. FASEB J 18: 1060-1070, 2004.

2. Widmann C, Gibson S, Jarpe MB and Johnson GL: Mitogenactivated protein kinase: conservation of a three-kinase module from yeast to human. Physiol Rev 79: 143-180, 1999.

3. Tournier C, Whitmarsh AJ, Cavanagh J, Barrett T and Davis RJ: Mitogen-activated protein kinase kinase 7 is an activator of the c-Jun NH2-terminal kinase. Proc Natl Acad Sci USA 94: 7337-7342, 1997.

4. Barr RK and Bogoyevitch MA: The c-Jun N-terminal protein kinase family of mitogen-activated protein kinases (JNK MAPKs). Int J Biochem Cell Biol 33: 1047-1063, 2001.

5. Davis RJ: Signal transduction by the JNK group of MAP kinases. Cell 103: 239-252, 2000.

6. Chang L and Karin M: Mammalian MAP kinase signaling cascades. Nature 410: 37-40, 2001

7. Dunn C, Wiltshire C, MacLaren A and Gillespie DA: Molecular mechanism and biological functions of c-Jun N-terminal kinase signalling via the c-Jun transcription factor. Cell Signal 14: 585-593, 2002.

8. Xia Z, Dickens M, Raingeaud J, Davis RJ and Greenberg ME: Opposing effects of ERK and JNK-p38 MAP kinases on apoptosis. Science 270: 1326-1331, 1995.

9. Liu J and Lin A: Role of JNK activation in apoptosis: a doubleedged sword. Cell Res 15: 36-42, 2005.

10. Orstavik S, Natarajan V, Taskén K, Jahnsen T and Sandberg M: Characterization of the human gene encoding the type Ialpha and type Ibeta cGMP-dependent protein kinase (PRKG1). Genomics 42: 311-318, 1997.

11. Orstavik S, Solberg R, Taskén K, et al: Molecular cloning, cDNAstructure and chromosomal localization of the human type IIcGMP-dependent protein kinase. Biochem Biophys Res Commun 220: 759-765, 1996.

12. Browing DD: Protein kinase G as a therapeutic target for the treatment of metastatic colorectal cancer. Expert Opin Ther Targets 12: 367-376, 2008

13. Chen YC, Ren F, Sang JR, Tao Y and Xu WX: Type II cGMPdependent protein kinase inhibits proliferation of the gastric cancer cell line BGC-823. Mol Med Rep 3: 361-366, 2010.

14. Wu Y, Chen YC, Qu R, Lan T and Sang JR: Type II cGMP-dependent protein kinase inhibits EGF-triggered signal transduction of the MAPK/ERK-mediated pathway in gastric cancer cells. Oncol Rep 27: 553-558, 2012.

15. Jiang H, Grenley MO, Bravo MJ, et al: EGFR/Ras/MAPK signaling mediates adult midgut epithelial homeostasis and regeneration in Drosophila. Cell Stem Cell 8: 84-95, 2011.

16. de Rooij J and Bos JL: Minimal Ras-binding domain of Raf 1 can be used as an activation-specific probe for Ras. Oncogene 14: 623-625, 1997.

17. Hofmann F: The biology of cyclic GMP-dependent protein kinases. J Biol Chem 280: 1-4, 2005.

18. Nie HG, Chen L, Han DY, et al: Regulation of epithelial sodium channels by cGMP/PKGII. J Physiol 587: 2663-2676, 2009.

19. Rangaswami H, Marathe N, Zhuang S, Chen Y, Yeh JC, Frangos JA, Boss GR and Pilz RB: Type II cGMP-dependent protein kinase mediates osteoblast mechanotransduction. J Biol Chem 284: 14796-14808, 2009 
20. Rangaswami H, Schwappacher R, Marathe N, et al: Cyclic GMP and protein kinase $\mathrm{G}$ control a Src-containing mechanosome in osteoblasts. Sci Signal 3: ra91, 2010.

21. Cook AL and Haynes JM: Protein kinase G II-mediated proliferative effects in human cultured prostatic stromal cells. Cel Signal 16: 253-261, 2004.

22. Swartling FJ, Ferletta M, Kastemar M, Weiss WA and Westermark B: Cyclic GMP-dependent protein kinase II inhibits cell proliferation, Sox 9 expression and Akt phosphorylation in human glioma cell lines. Oncogene 28: 3121-3131, 2009.

23. Fallahian F, Karami-Tehrani F, Salami S and Aghaei M: Cyclic GMP induced apoptosis via protein kinase $\mathrm{G}$ in oestrogen receptorpositive and -negative breast cancer cell lines. FEBS J 278: $3360-3369,2011$

24. Wells A: EGF receptor. Int J Biochem Cell Biol 31: 637-643, 1999.
25. Saito T, Okada S, Ohshima K, et al: Differential activation of epidermal growth factor (EGF) receptor downstream signaling pathways by betacellulin and EGF. Endocrinology 145: 4232-4243, 2004.

26. Hynes NE and MacDonald G: ErbB receptors and signaling pathways in cancer. Curr Opin Cell Biol 21: 177-184, 2009.

27. Normanno N, Bianco C, De Luca A, et al: Target-based agents against ErbB receptors and their ligands: a novel approach to cancer treatment. Endocr Relat Cancer 10: 1-21, 2003.

28. Sharma SV, Bell DW, Settleman J, et al: Epidermal growth factor receptor mutations in lung cancer. Nat Rev Cancer 7: 169-181, 2007.

29. Hu T and Li C: Convergence between Wnt- $\beta$-catenin and EGFR signaling in cancer. Mol Cancer 9: 236, 2010.

30. Quatrale AE, Porcelli L, Silvestris N, Colucci G, Angelo A and Azzariti A: EGFR tyrosine kinases inhibitors in cancer treatment: in vitro and in vivo evidence (Review). Front Biosci 16: 1962-1972, 2011. 